\title{
Sarennes, un bassin versant de recherche et d'expérimentation nivo-glaciaire, cinquante années d'études
}

\author{
Sarennnes, a catchment area for nivo-glacial research and experiments : \\ fifty years of studies \\ par François Valla, \\ Cemagref, division Erosion torrentielle neige et avalanches
}

The basin of Sarennes is located in the French Alps, more precisely in the Grande Rousses range, $30 \mathrm{~km}$ east of Grenoble. The catchment area reaches some 30 square kilometers with a small glacier studied since 1948. Hydrologic studies started in 1991 with the installation of 3 gauging stations at $2720 \mathrm{~m}$., $2050 \mathrm{~m}$. and 1450 meters of altitude. An automatic snow gauge called "telenivometer" and a simplified meteorological station were installed close to the laboratory-hut at the altitude of 2700 m., nearby the glacier tongue. In 1991, the Sarennes basin was selected for the ERB club (European Research Basin).

This paper provides a description of this basin unique in France due to its glacial and snowy components. Mass balance evolution for the last half century shows an annual loss of 0.6 meters of water equivalent and we present the first hydrologic results and the ultimate results of the field $1998-99$ campaign.

\section{PRÉSENTATION DU BASSIN VERSANT}

Le bassin de Sarennes est situé dans les Alpes françaises, 30 kilomètres à l'Est de Grenoble, dans le domaine actuel de la station de ski de l'Alpe d'Huez. L'ensemble du bassin versant couvre $28,2 \mathrm{~km}^{2}$ et se décompose en trois sous-bassins emboîtés d'altitude décroissante.

\subsection{Le bassin supérieur}

C'est le site du glacier de Sarennes. Il est occupé principalement par un petit glacier de cirque, relique de la dernière extension glaciaire et a retenu l'attention des glaciologues depuis plus d'un siècle. Visité par le naturaliste Roland Bonaparte en 1891, il fut remarquablement cartographié en 1905-1906 (surface de $1,08 \mathrm{~km}^{2}$ ) par trois universitaires de Grenoble travaillant sur le massif de Grandes Rousses [1]. Réduit de moitié en près d'un siècle, il occupe actuellement une surface de $0,5 \mathrm{~km}^{2}$. Situé sous le $45^{\circ}$ parallèle $\left(45^{\circ} 6^{\prime} \mathrm{N}\right.$, $\left.6^{\circ} 8^{\prime} \mathrm{E}\right)$, il est exposé au Sud, ce qui le rend sensible à l'ablation automnale. Il se développe entre 3200 et 2800 mètres d'altitude et son épaisseur maximale mesurée par radar (Funk, Bösch, Valla, 1993) est d'environ 80 mètres. La surface totale du bassin glaciaire est de $1,3 \mathrm{~km}^{2}$ (altitude 3323 $2700 \mathrm{~m}$ ), la contribution de la surface du glacier diminuant au cours du temps.

\subsection{Le bassin médian}

Il comprend le bassin supérieur et représente une surface totale de $5 \mathrm{~km}^{2}$, comprise entre 3323 et 2050 mètres d'une orientation générale Sud. Il correspond à la vallée supérieure du torrent de la Sarennes dont le régime est essentiellement nival. En dehors de l'émissaire issu du glacier, seuls de petits ruisseaux contribuent à son alimentation.

\subsection{Le bassin global}

Sa surface totale est de $28,2 \mathrm{~km}^{2}$ et ce bassin recouvre la majeure partie du domaine skiable de l'Alpe d'Huez. Sa limite inférieure est à 1450 mètres, dans les gorges de Sarennes au lieu-dit "Les Moulins" (anciens moulins à grain actionnés par le torrent), après la confluence avec les eaux issues du Lac Blanc. Sa délimitation suit les lignes de crête suivantes : La Grande Sure $(2114 \mathrm{~m})$, Col de Poutran (2006 m), Dôme des Petites Rousses (2810 m), Col du Lac Blanc (2740 m), Col du Milieu (3256 m), Pic du Lac Blanc (3323 m), Sommets Nord et Sud de Sarennes (3129 et 3063 m), Château Noir (2882 m), Col de Sarennes (1999 m), La Croix de Cassini (2373 m), Les Grandes Buffes $(2164 \mathrm{~m})$, Signal de l'Homme $(2176 \mathrm{~m})$ et traverse le village de l'Alpe d'Huez en son milieu (figure 1).

La répartition par tranche d'altitude est la suivante : $11,3 \mathrm{~km}^{2}(40,0 \%)$ entre 1450 et 2000 mètres, $8,0 \mathrm{~km}^{2}$ $(28,4 \%)$ entre 2000 et 2500 mètres, $7,4 \mathrm{~km}^{2}(26,2 \%)$ entre 


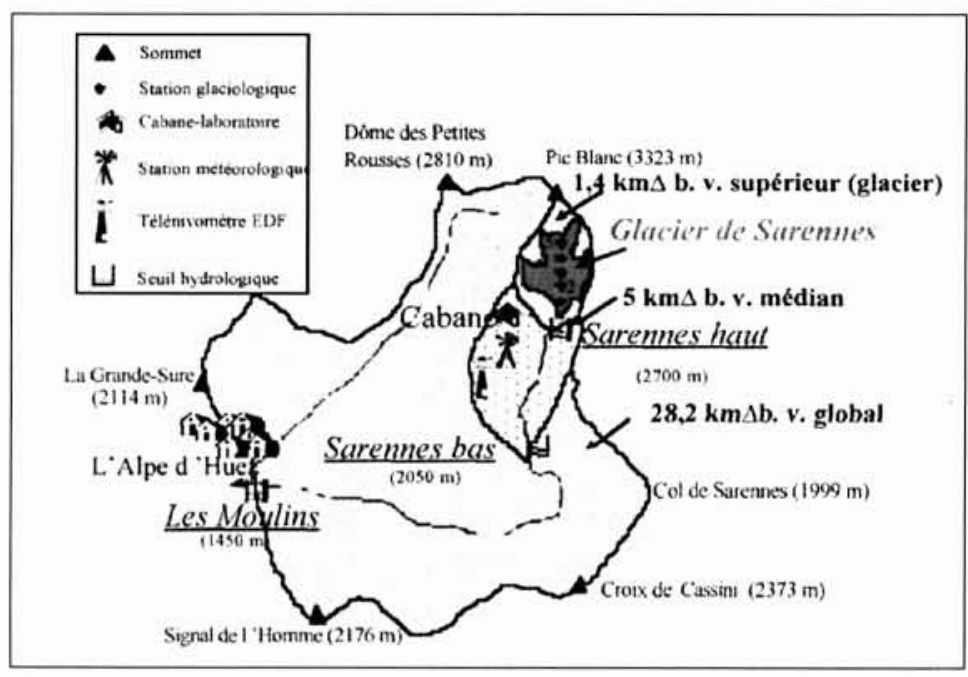

1. Le BVRE Sarennes et ses équipements.

lents en eau relevés en 5 stations étagées de 3050 à 2800 mètres le long d'un profil longitudinal. Ce stock est suivi tout au long de la saison estivale par carottage jusqu'à l'apparition de la glace vive. La perte en glace est mesurée par lecture de balises d'ablation (perches de 10 mètres formées de 5 tronçons de couleurs différentes de 2 mètres) placées aux cinq stations. Le bilan global du glacier est obtenu en pondérant la mesure de chaque station par la surface qu'elle représente (35\% pour la station 5 qui est la plus élevée, $25 \%$ pour les 4 et $3,10 \%$ pour la station 2 et $5 \%$ pour la station 1 , la plus basse) :

- bilan global $($ jour $\mathrm{j})=($ mesure station $5 \mathrm{x}$ $0,35)+($ mes. st. $4 \times 0,25)+($ mes. st. $3 \times 0,25)+$ (mes. st. $2 \times 0,10)+($ mes. st. $1 \times 0,05)$

Le bilan de masse de l'année est mesuré à la fin de la saison d'ablation en principe autour du 30 septembre ou courant octobre si l'arrière-saison est ensoleillée. Au total, nous disposons de plus de 300 mesures de bilan qui décrivent l'évolution

2500 et 3000 mètres et $1,5 \mathrm{~km}^{2}(5,4 \%)$ entre 3000 et 3023 mètres. Notons enfin que le bassin supérieur représente environ $5 \%$ du global et le médian moins de $20 \%$.

\section{II $\square$ MESURES GLACIOLOGIQUES}

Depuis 1948-49 [2] le glacier de Sarennes est observé et mesuré chaque année, au cours de tournées estivales qui permettent de suivre l'évolution du bilan glaciaire. Le stock de neige hivernal est mesuré par carottage début juin, fournissant la valeur de l'accumulation à partir des hauteurs de neige (qui atteignent parfois 6 mètres), densités et équiva- du glacier depuis bientôt 50 ans [3]. La figure 2 présente l'évolution des principales données glaciologiques, accumulation, ablation, bilan de masse et régime de 1948 à 1997 ainsi que l'évolution au cours d'une saison estivale des paramètres suivis sur le glacier. La figure 3 montre l'évolution du bilan de masse cumulé depuis le début des mesures, courbe qui, compte tenu de l'absence de mouvement du glacier, visualise la baisse de niveau du glacier (la glace ayant pour densité 0,9 , la perte de 30 mètres d'eau représente une baisse du niveau du glacier de 33,5 mètres en 49 ans, soit une perte moyenne annuelle de 60 centimètres d'équivalent en eau ou $66 \mathrm{~cm}$ de glace.

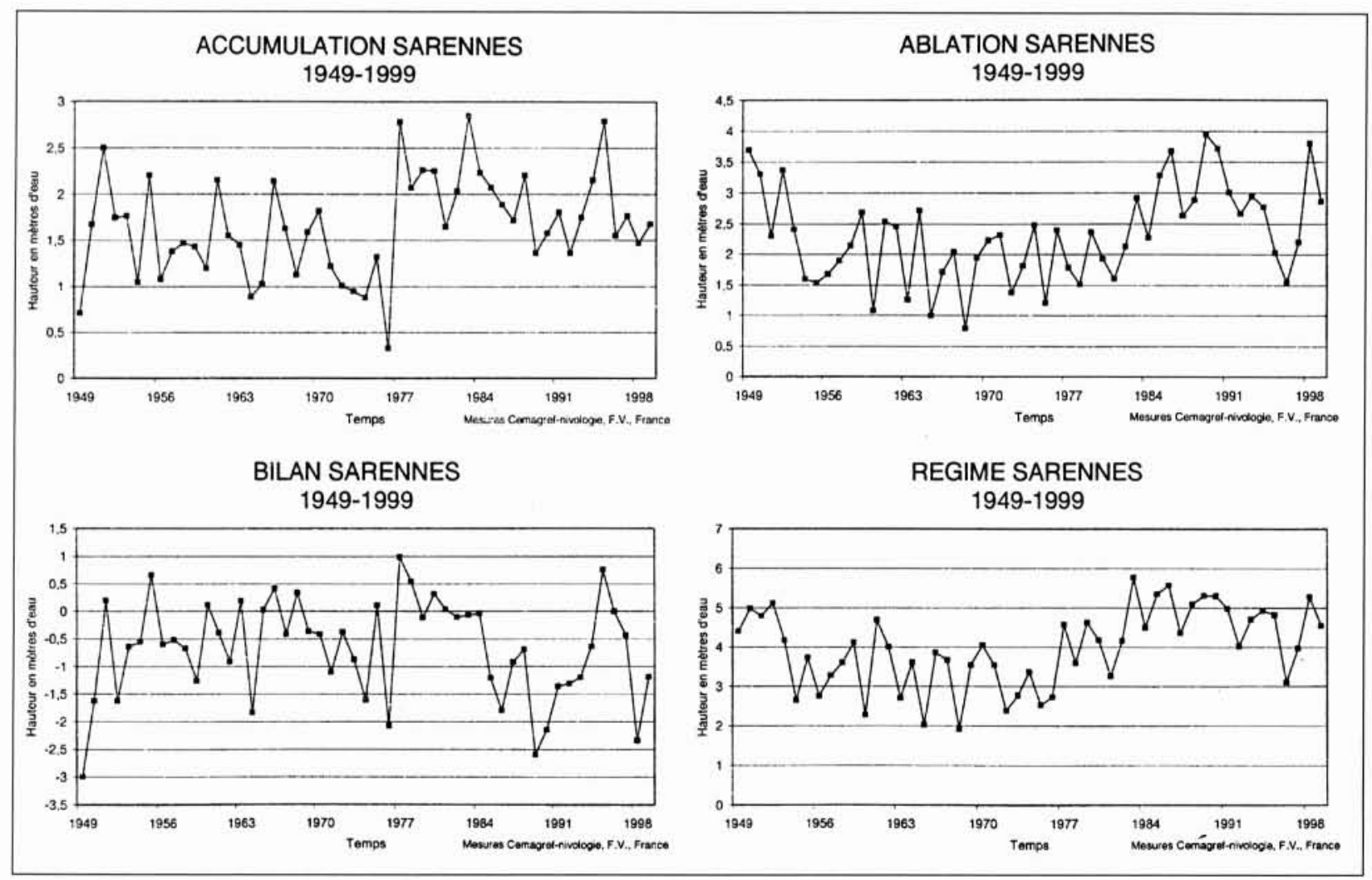

2. Données glaciologiques relatives au Glacier de Sarennes, 1948-99. 


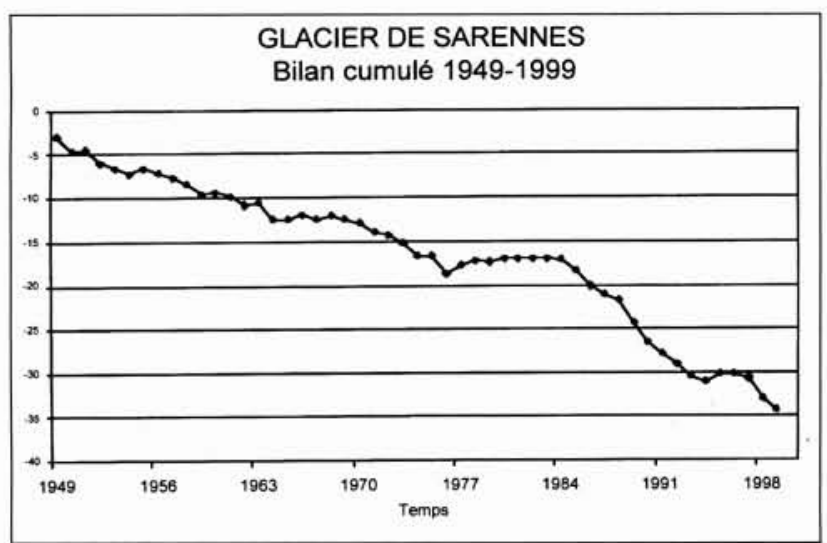

3. Evolution du bilan de masse cumulé du Glacier de Sarennes, 1948-99.

\section{III — EQUIPEMENT DU BVRE}

Nous distinguerons les équipements concernant l'étude du glacier de ceux, plus récents, mis en place dans le cadre du BVRE.

En 1950, (avant l'installation du téléphérique du Lac Blanc à $3300 \mathrm{~m}$ ), la cabane de Sarennes fut installée à 2740 mètres, légèrement en aval du glacier. Ce petit refuge en mélèze de 2 mètres sur 4 est toujours vaillant et nous sert aujourd'hui de "cabane-laboratoire". Une perche à neige de 6 mètres et un pluviomètre Mougin désaffecté datent de cette époque.

Dès 1977 le glacier a été équipé de grandes balises d'ablation fixes de 10 mètres implantées par forage aux 5 stations. Ce travail a nécessité le concours technique du Laboratoire de Glaciologie du CNRS de Grenoble (LGGE, Yves Morin, Louis Reynaud et Christian Vincent) et est à renouveler périodiquement.

En 1991, lorsque le site a accédé au rang de BVRE, un seuil hydrologique en béton a été installé sur l'émissaire du glacier, au niveau de la cabane, à 2700 mètres d'altitude. Deux capteurs, un de pression hydrostatique et l'autre de
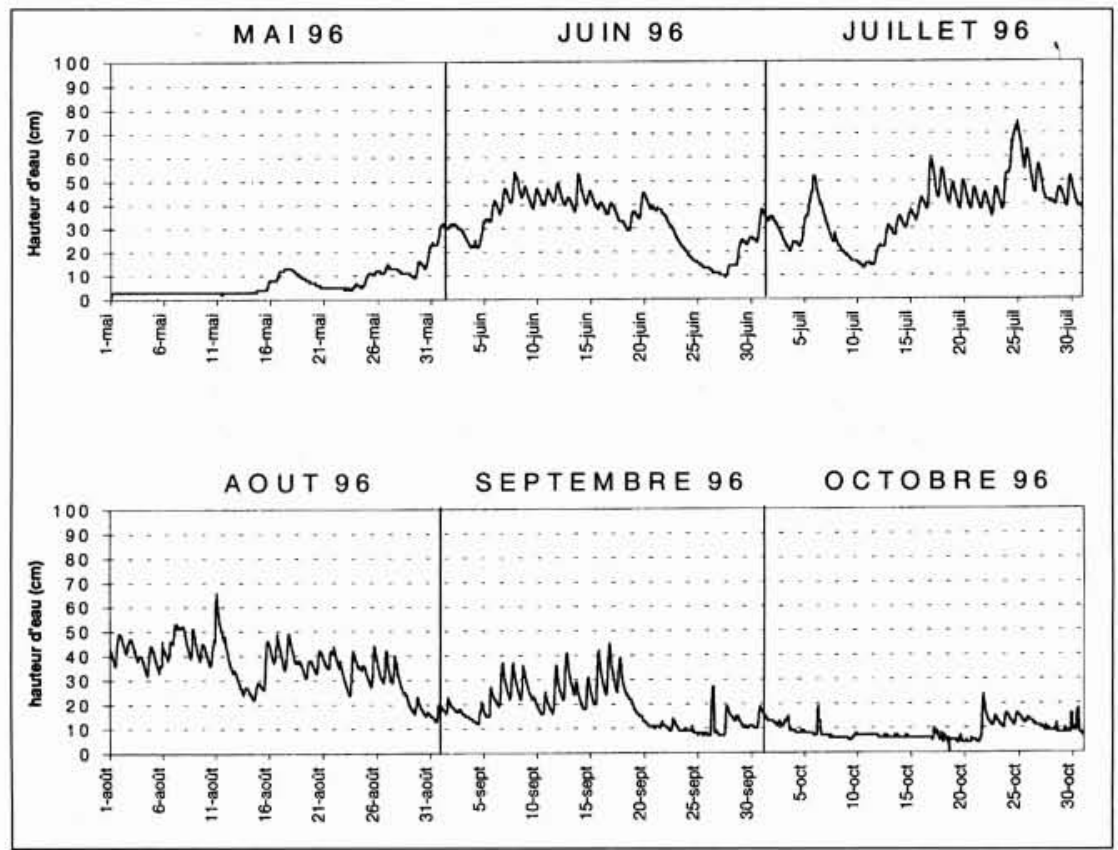

4. Hauteur d'eau à l'émissaire du glacier en 1996. capacité électrique, mesurent la hauteur d'eau et sont enregistrés par une centrale Unidata (scrutation toutes les 5 secondes et enregistrement de la valeur moyenne toutes les 10 minutes). Ce système a été doublé l'année suivante afin d'assurer une fiabilité de la mesure maximale, le seuil étant d'accès très difficile pendant plus de 8 mois car recouvert de 5 à 6 mètres de neige. Pour contrôler les centrales et les décharger pendant l'hiver, il faut maintenir un puits de visite dans le manteau neigeux.

En 1993, grâce à un financement BVRE, un télénivomètre EDF horizontal a été mis en service à 2730 mètres près de la cabane. Cet appareil, muni d'une source radioactive de Césium 137 (10 millicurie), relève journellement la hauteur de neige et donne densité et équivalent en eau du manteau neigeux par couches de $10 \mathrm{~cm}$.

Un second seuil appelé "Sarennes Bas" a été mis en service à 2050 mètres sur le cours moyen du torrent en 1994. Il est aussi équipé de deux capteurs de hauteur d'eau (capacitif et pression) enregistrés par une centrale de mesure Unidata.

Le troisième seuil a été réalisé par EDF en 1976 à la cote 1450 mètres, et récolte aussi les eaux issues du Lac Blanc. Il est équipé d'un limnigraphe enregistreur et est géré par EDF, Direction de Techniques Générales de Grenoble.

Enfin une petite station météorologique enregistrant les températures (instantanée, maximum et minimum) ainsi que le vent (intensité et direction), le rayonnement incident et les précipitations liquides est en cours d'installation à la cabanelaboratoire. Notons qu'une station mobile (température, vent et précipitations) a été mise en place l'été 1996 sur le glacier avec succès mais demande une surveillance constante à cause de l'ablation qui peut atteindre 5 centimètres par jour et déséquilibre le mât portant les capteurs [4].

L'équipement actuellement en place pourrait être complété par des mesures de précipitations en plusieurs points du bassin versant, des mesures de rayonnement et d'albédo ainsi que d'évaporation en vue d'établir un bilan d'énergie. L'exemple du suivi des bilans glaciologique, hydrologique et énergétique du Glacier Zongo (Bolivie) pourrait nous servir de modèle [5].

Enfin, signalons l'utilisation de SIG (Système d'Informations Géographiques) qui a permis de stocker sur une base de données la totalité des mesures spatiotemporelles glaciologiques [6] et de calculer la perte volumétrique du glacier depuis les toutes premières observations [7].

\section{IV — MESURES HYDROLO- GIQUES}

Les trois seuils hydrologiques, Sarennes Haut $(2700 \mathrm{~m})$, Sarennes Bas (2050 m) et Les Moulins (1450 m., géré par EDF) enregistrent les hauteurs d'eau en continu. Des campagnes de jaugeage ont permis d'établir les courbes de tarage relatives, avec toutes les difficultés liées à l'exploitation de site de montagne et de haute montagne. Les chroniques sont disponibles :

- au pas de temps de 10 minutes pour Sarennes Haut à partir de 1992 . 

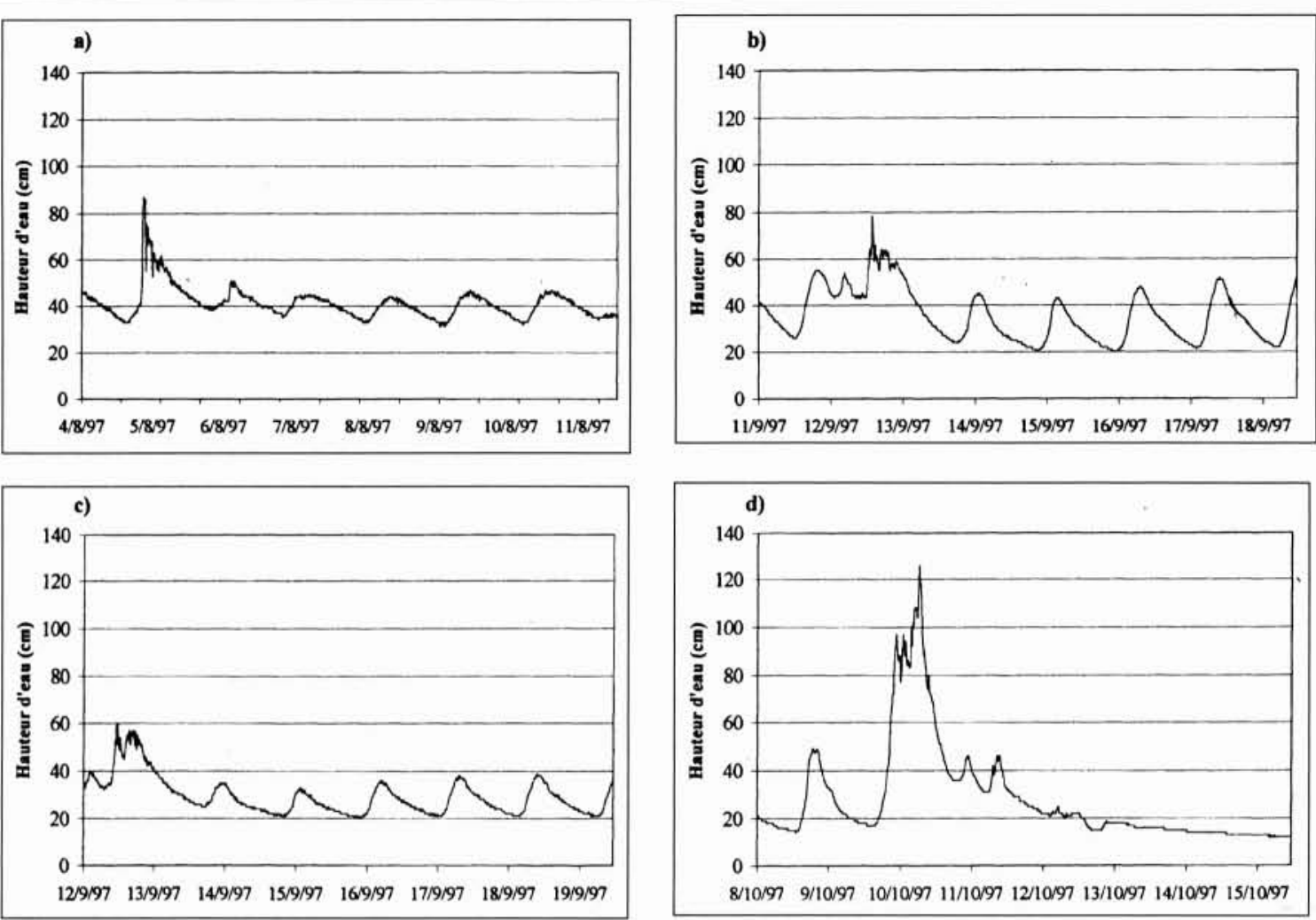

a) Seuil du Bas, 5-10 aout 97 : orage le 5 sur le bassin versant médian, modulant fortement l'apport journalier issu du glacier encore enneigé (faible amplitude journalière).

b) Seuil du Haut, le-30 septembre 97 : fonte glaciaire active avec précipitations le 14.

c) Seuil du Bas, 13-15 septembre 97 : pluie et neige en altitude avec fonte dans la journée.

d) Seuil du Haut, 8-29 octobre 97 : très violente crue le 10, pluie au dessus de 3000.

\section{Hydrogrammes de l'été 1997, seuils Sarennes Haut et Sarennes Bas.}

- au pas de temps de 10 minutes pour Sarennes Bas à partir de 1994

- et au pas de temps horaire pour Les Moulins dès fin 1976 (enregistrement papier).

La figure 4 présente la variation de la hauteur d'eau au seuil Sarennes Haut pour les six mois de la période maioctobre 1996, la courbe de tarage étant en cours de validation. Les premières analyses [8] montrent pour le bassin versant glaciaire trois types de régimes caractéristiques.

Le régime hivernal est absolument constant pendant les mois d'hiver, généralement de novembre à mars ou avril. Toutes les précipitations tombent sous forme solide ou sont piégées par le manteau neigeux. Le flux géothermique est seul responsable du débit liquide passant au seuil à l'altitude de 2700 mètres (une dizaine de litres par seconde).

Le régime nival apparaît dès que la température diurne commence à faire fondre la neige. Comme le bassin versant est couvert d'un épais manteau neigeux, la fonte met plusieurs jours à imprégner la neige et à percoler jusqu'à la couche de glace imperméable qui va drainer cette eau de fonte. L'hydrogramme présente donc un niveau permanent d'écoulement élevé ( 30 à $40 \mathrm{~cm}$ de hauteur, soit dans les 200 litres par seconde) qui est modulé journellement avec un fort déphasage, fonction de l'épaisseur du manteau neigeux. Ce régime perdure jusqu'en août dans le diagramme présenté.

Le régime purement glaciaire apparaît lorsque le glacier est mis à nu, généralement en août. L'amplitude des oscillations journalières est plus importante, le déphasage se réduit à quelques heures et le niveau d'écoulement nocturne est moins élevé (20 cm d'eau, soit moins de 100 litres par seconde).

A partir de septembre et octobre, on note les arrivées de précipitations hivernales qui bloquent la fonte, le glacier étant alors recouvert de neige fraîche stoppant net l'écoulement. Les périodes de réchauffement automnale sont marquées par de petites variations journalières de faible amplitude (fin septembre et mi octobre).

La figure 5 présente quelques exemples d'hydrogrammes de l'été 1997.

\section{V — LA CAMPAGNE DE 1998-1999}

\subsection{Les données météorologiques}

Rappelons que les courbes de températures et précipitations mensuelles sont celles de Grenoble St Martin d'Hères. Bien que situé à 212 mètres d'altitude, ce poste météorologique permet de se faire une bonne idée des conditions qui règnent au Glacier de Sarennes et présente une série complète. Une petite station météorologique a été récemment implantée à l'altitude de 2700 mètres, reliée à la cabanelaboratoire de Sarennes et les premiers enregistrements sont en cours de traitement. 


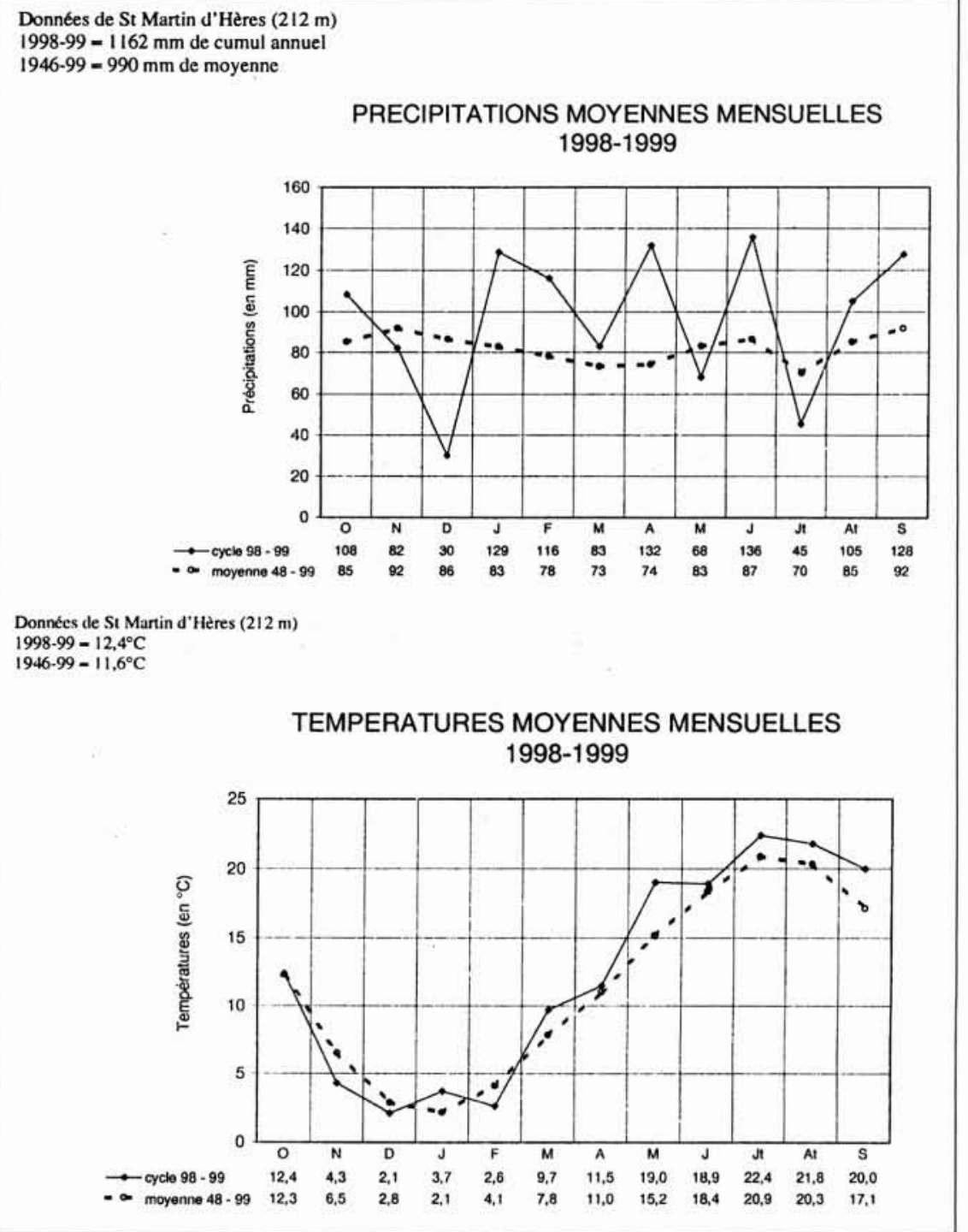

6. Données météorologiques de St Martin d'Hères.

Depuis 10 ans nous subissons des années anormalement chaudes, et l'été 99 fut encore plus chaud que le précédent. Cet excédent de chaleur de juin à septembre a été en partie compensé par les basses températures qui ont accompagné les fortes chutes de neige de l'hiver, si bien que la moyenne annuelle 1999 excède la moyenne 1946 1999 de $0,8^{\circ} \mathrm{C}$

Les précipitations cette année ont été excédentaires avec un cumul annuel de $1162 \mathrm{~mm}$ d'eau pour une moyenne de $990 \mathrm{~mm}$ sur la période 1946-99. La contribution des précipitations hivernales est importante (116 mm en février contre $78 \mathrm{~mm}$ de moyenne depuis 1948).

Le total hivernal octobre-mai dépasse de $100 \mathrm{~mm}$ l'apport moyen hivernal qui est pour les 51 dernières années de $655 \mathrm{~mm}$. Les précipitations estivales excèdent elles aussi la moyenne sur les 51 années de suivi, mais dans une moindre mesure.

\subsection{La campagne de mesure}

Les mesures de la campagne d'été se sont échelonnées du 7 mai au 6 octobre, date à laquelle sont apparues les premières chutes de neige. Les tableaux sont présentés ci-après ainsi que la marche de l'ablation.

\subsection{Les caractéristiques du cycle 1998-1999}

L'accumulation a été maximale au début du mois de juin, suite à une série d'épisodes neigeux qui ont augmenté l'accumulation relevée en mai d'environ 1 mètre d'Eqe sur l'ensemble du glacier. L'été a connu des températures élevées, portant l'ablation totale à 2,87 mètres, ce qui reste en deçà des valeurs de l'été précédent mais place le 51 ème cycle au 13ème rang des années les plus défavorables au glacier.

Le bilan est le 16ème le plus négatif avec $-1,19 \mathrm{mEqe}$ (moyenne à 0,67 ) et le régime le 33 ème le plus fort avec 4,55 mEqe (moyenne à 3,98 ).

Les valeurs successives relatives aux mesures des 5 stations jalonnant le glacier sont données par les tableaux (l'évolution des hauteurs de neige, des équivalents en eau, des densités et des vitesses d'ablation pour chacune des cinq stations du glacier de Sarennes, entre deux sorties sur le terrain).

En conclusion, on peut dire que la saison 1998-1999 est une année humide et chaude. Les précipitations, hivernales comme estivales, ont permis de ne pas assister à une ablation galopante, comme ce fut le cas l'année

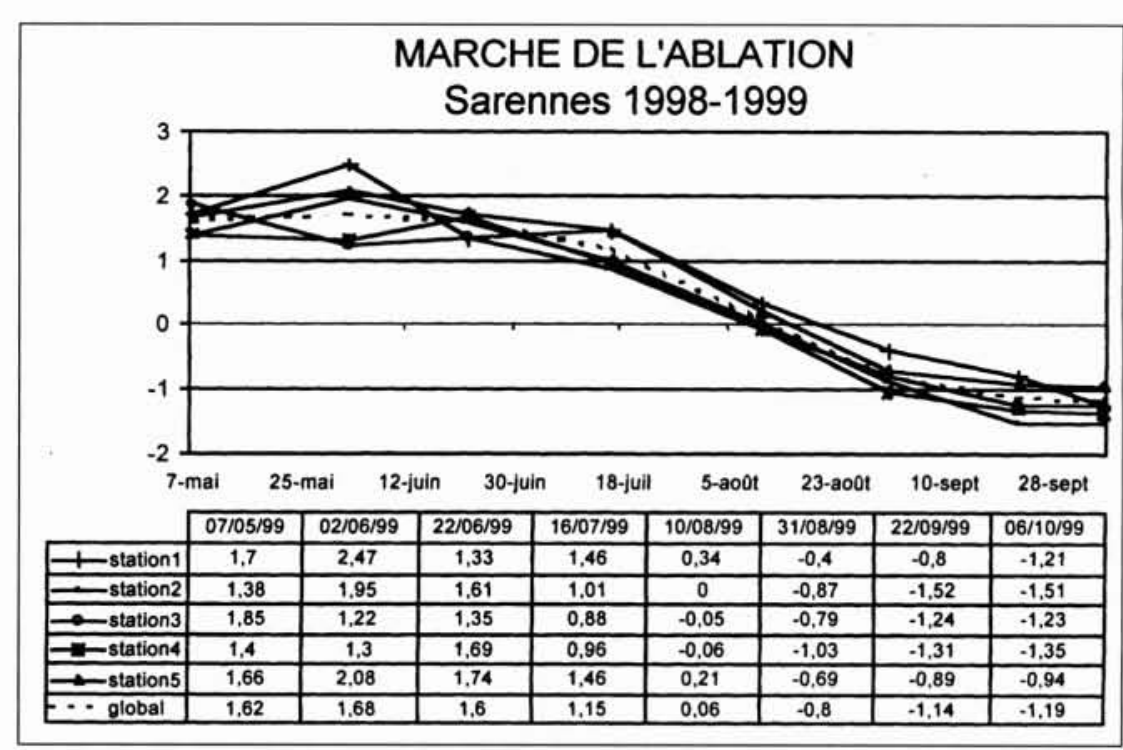

7. Evolution du stock neigeux et marche de l'ablation. 


\begin{tabular}{|c|c|c|c|c|c|}
\hline cycle & année & accumulation & ablation & bilan & régime \\
\hline 1 & $1948-49$ & 0,71 & 3,70 & $-2,99$ & 4,41 \\
\hline 2 & $49-50$ & 1,68 & 3,30 & $-1,62$ & 4,98 \\
\hline 3 & $1950-51$ & 2,50 & 2.30 & $+0,20$ & 4,80 \\
\hline 4 & $51-52$ & 1,75 & 3,37 & $-1,62$ & 5,12 \\
\hline 5 & $52-53$ & 1,77 & 2,41 & $-0,64$ & 4,18 \\
\hline 6 & $53-54$ & 1,05 & 1,60 & $-0,55$ & 2,65 \\
\hline 7 & $54-55$ & 2,20 & 1,54 & $+0,66$ & 3,74 \\
\hline 8 & $55-56$ & 1,08 & 1,68 & $-0,60$ & 2,76 \\
\hline 9 & $56-57$ & 1,38 & 1,90 & $-0,52$ & 3,28 \\
\hline 10 & $57-58$ & 1,47 & 2,14 & $-0,67$ & 3,61 \\
\hline 11 & $58-59$ & 1,43 & 2,69 & $-1,26$ & 4,12 \\
\hline 12 & $59-60$ & 1,20 & 1,08 & $+0,12$ & 2,28 \\
\hline 13 & $1960-61$ & 2,15 & 2,54 & $-0,39$ & 4,69 \\
\hline 14 & $61-62$ & 1,55 & 2,46 & $-0,91$ & 4,01 \\
\hline 15 & $62-63$ & 1,45 & 1,26 & $+0,19$ & 2,71 \\
\hline 16 & $63-64$ & 0,89 & 2,72 & $-1,83$ & 3,61 \\
\hline 17 & $64-65$ & 1,03 & 1,00 & $+0,03$ & 2,03 \\
\hline 18 & $65-66$ & 2,14 & 1,72 & $+0,42$ & 3,86 \\
\hline 19 & $66-67$ & 1,63 & 2,04 & $-0,41$ & 3,67 \\
\hline 20 & $67-68$ & 1,13 & 0,79 & $+0,34$ & 1,92 \\
\hline 21 & $68-69$ & 1,59 & 1,95 & $-0,36$ & 3,54 \\
\hline 22 & $69-70$ & 1,82 & 2,23 & $-0,41$ & 4,05 \\
\hline 23 & $1970-71$ & 1,22 & 2,32 & $-1,10$ & 3,54 \\
\hline 24 & $71-72$ & 1,01 & 1,38 & $-0,37$ & 2,39 \\
\hline 25 & $72-73$ & 0,95 & 1,82 & $-0,87$ & 2,77 \\
\hline 26 & $73-74$ & 0,88 & 2,48 & $-1,60$ & 3,36 \\
\hline 27 & $74-75$ & 1,32 & 1,21 & $+0,11$ & 2,53 \\
\hline 28 & $75-76$ & 0,33 & 2,40 & $-2,07$ & 2,73 \\
\hline 29 & $76-77$ & 2,78 & 1,79 & $+0,99$ & 4,57 \\
\hline 30 & $77-78$ & 2,07 & 1,52 & $+0,55$ & 3,59 \\
\hline 31 & $78-79$ & 2,26 & 2,37 & $-0,11$ & 4,63 \\
\hline 32 & $79-80$ & 2,25 & 1,93 & $+0,32$ & 4,18 \\
\hline 33 & $1980-81$ & 1,65 & 1,61 & $+0,04$ & 3,26 \\
\hline 34 & $81-82$ & 2,03 & 2,13 & $-0,10$ & 4,16 \\
\hline 35 & $82-83$ & 2,85 & 2,92 & $-0,07$ & 5,77 \\
\hline 36 & $83-84$ & 2,23 & 2,27 & $-0,04$ & 4,50 \\
\hline 37 & $84-85$ & 2,07 & 3,28 & $-1,21$ & 5,35 \\
\hline 38 & $85-86$ & 1,89 & 3,68 & $-1,79$ & 5,57 \\
\hline 39 & $86-87$ & 1,72 & 2,64 & $-0,92$ & 4,36 \\
\hline 40 & $87-88$ & 2,20 & 2,89 & $-0,69$ & 5,09 \\
\hline 41 & $88-89$ & 1,36 & 3,95 & $-2,59$ & 5,31 \\
\hline 42 & $89-90$ & 1,58 & 3,72 & $-2,14$ & 5,30 \\
\hline 43 & $90-91$ & 1,81 & 3,01 & $-1,20$ & 4,81 \\
\hline 44 & $91-92$ & 1,36 & 2,67 & $-1,31$ & 4,03 \\
\hline 45 & $92-93$ & 1,75 & 2,95 & $-1,20$ & 4,70 \\
\hline 46 & $93-94$ & 2,15 & 2,78 & $-0,63$ & 4,93 \\
\hline 47 & $94-95$ & 2,79 & 2,03 & $+0,76$ & 4,82 \\
\hline 48 & $95-96$ & 1,55 & 1,55 & 0 & 3,10 \\
\hline 49 & $96-97$ & 1,77 & 2,21 & $-0,50$ & 4,04 \\
\hline 50 & $97-98$ & 1,47 & 3,81 & $-2,34$ & 5,28 \\
\hline 51 & 98-99 & 1,68 & 2,87 & $-1,19$ & 4,45 \\
\hline
\end{tabular}




\begin{tabular}{|c|c|c|c|c|c|c|c|c|c|c|c|c|c|c|c|c|c|c|c|c|}
\hline \multirow[b]{2}{*}{ date } & \multirow{2}{*}{ Haut. } & \multicolumn{3}{|c|}{ Station 1} & \multicolumn{4}{|c|}{ Station 2} & \multicolumn{4}{|c|}{ Station 3} & \multicolumn{4}{|c|}{ Station 4} & \multicolumn{4}{|c|}{ Station 5} \\
\hline & & Eqe & |Dens & Vabl & Haut. & Eqe & Dens & Vabl & Haut. & Eqe & dens & Vabl & Haut. & Eqe & dens & Vabl & Haut. & Eqe & dens & Vabl \\
\hline $7 / 5 / 99$ & 3,80 & 1,70 & 447 & & 3,07 & 1,38 & 450 & & 3,98 & 1,85 & 465 & & 3,12 & 1,40 & 449 & & 3,97 & 1,66 & 418 & \\
\hline $2 / 6 / 99$ & 4,60 & 2,47 & 537 & $-3,0$ & 3,54 & 1,95 & 551 & $-2,2$ & 2,76 & 1,22 & 442 & 2,4 & 2,84 & 1,30 & 458 & 0,4 & 3,85 & 2,08 & 540 & $-1,6$ \\
\hline $22 / 6 / 99$ & 2,40 & 1,33 & 554 & 5,7 & 2,94 & 1,61 & 548 & 1,7 & 2,52 & 1,35 & 536 & $-0,7$ & 3,06 & 1,69 & 552 & $-1,9$ & 3,30 & 1,74 & 527 & 1,7 \\
\hline $16 / 7 / 99$ & 2,43 & 1,46 & 601 & $-0,5$ & 1,62 & 1,01 & 623 & 2,5 & 1,46 & 0,88 & 603 & 2,0 & 1,60 & 0,96 & 600 & 3,0 & 2,45 & 1,46 & 596 & 1,2 \\
\hline $10 / 8 / 99$ & 0,50 & 0,34 & 676 & 4,5 & & 0,00 & & 4,0 & & $-0,05$ & & 3,7 & & $-0,06$ & & 4,1 & & 0,21 & & 5,0 \\
\hline $31 / 8 / 99$ & & $-0,40$ & & 3,5 & & $-0,87$ & & 4,1 & & $-0,79$ & & 3,5 & & $-1,03$ & & 4,6 & & $-0,69$ & & 4,3 \\
\hline $22 / 9 / 99$ & & $-0,80$ & & 1,8 & & $-1,52$ & & 3,0 & & $-1,24$ & & 2,0 & & $-1,31$ & & 1,3 & & $-0,89$ & & 1,8 \\
\hline $6 / 10 / 99$ & & $-1,21$ & & 2,9 & & $-1,51$ & & $-0,1$ & & $-1,23$ & & $-0,1$ & & $-1,35$ & & 0,3 & & $-0,94$ & & $-1,0$ \\
\hline
\end{tabular}

\section{Campagne de mesures de l'année 1999}

\begin{tabular}{|c|c|c|c|c|c|c|c|c|c|c|}
\cline { 2 - 10 } \multicolumn{1}{c|}{} & Accumulation & periode & ablation & Vitesse abl & \multicolumn{4}{c|}{ Bilan par station } & \multicolumn{1}{c|}{ Bilan } \\
\hline date & (m Eqe.neige) & (jours) & (m Eqe) & cm/jour & sta1 & sta2 & sta3 & sta4 & sta5 & global \\
\hline $7 / 5 / 99$ & 1,62 & & & & 1,70 & 1,38 & 1,85 & 1,40 & 1,66 & 1,62 \\
\hline $2 / 6 / 99$ & 1,68 & 26 & $-0,06$ & $-0,2$ & 2,47 & 1,95 & 1,22 & 1,30 & 2,08 & 1,68 \\
\hline $22 / 6 / 99$ & 1,60 & 20 & 0,08 & 0,4 & 1,33 & 1,61 & 1,35 & 1,69 & 1,74 & 1,60 \\
\hline $16 / 7 / 99$ & 1,15 & 24 & 0,45 & 1,9 & 1,46 & 1,01 & 0,88 & 0,96 & 1,46 & 1,15 \\
\hline $10 / 8 / 99$ & 0,06 & 25 & 1,09 & 4,4 & 0,34 & 0,00 & $-0,05$ & $-0,06$ & 0,21 & 0,06 \\
\hline $31 / 8 / 99$ & & 21 & 0,86 & 4,1 & $-0,40$ & $-0,87$ & $-0,79$ & $-1,03$ & $-0,69$ & $-0,80$ \\
\hline $22 / 9 / 99$ & & 22 & 0,34 & 1,5 & $-0,80$ & $-1,52$ & $-1,24$ & $-1,31$ & $-0,89$ & $-1,14$ \\
\hline $6 / 10 / 99$ & & 14 & 0,05 & 0,4 & $-1,21$ & $-1,51$ & $-1,23$ & $-1,35$ & $-0,94$ & $-1,19$ \\
\hline
\end{tabular}

* toutes les valeurs sont en mètres Eqe (mètre équivalent en eau)

Ablation totale:

Bilan global : $\quad-1,19$

précédente. Depuis le cycle 1987-88, toutes les années ont eu une moyenne supérieure à $12^{\circ} \mathrm{C}$. Des étés spécialement ensoleillés ont provoqué des fontes excessives qui apparaissent sur la courbe du bilan cumulé du glacier de Sarennes, courbe dont la pente moyenne s'accentue depuis 15 ans.

\section{CONCLUSION}

Le Bassin Versant de Recherche et d'Expérimentation de Sarennes s'avère être un instrument de travail unique en France pour les hydrologues, nivologues et glaciologues. En Europe, seule l'Allemagne détient dans l'Oetztal (Alpes autrichiennes) un bassin glaciaire qui est étudié depuis 1973 (Escher-Vetter, Reinwarth, 1994). Avec bientôt cinquante années de mesures interrompues de bilans locaux à cinq altitudes différentes suivis pendant les 4 mois de la saison d'ablation ( 7 à 10 relevés annuels par station, soit environ 2500 points de mesure), avec des chroniques hydrologiques journalières à trois cotes d'altitude du torrent, le BVRE et sa base de données intéressent tout à la fois les hydro-nivologues $[9,10]$ et les glaciologues [11]. Les travaux publiés en témoignent.

Le soutien financier du Conseil Scientifique et Technique des BVRE permet enfin d'envisager l'avenir du suivi de ce site tant du point de vue des mesures collectées que de leur valorisation par la communauté scientifique nationale et internationale.

\section{Remerciements}

L'auteur souhaite associer à cet article et remercier tous ceux qui ont participé, au cours de ce demi-siècle, au suivi du glacier de Sarennes. D'abord les pionniers, Messieurs Cherrey, Garavel et de Crécy, les organismes gestionnaires, Eaux et Forêts, puis Cerafer, Ctgref et maintenant Cemagref, la SATA, le CNRS-LGGE en la personne de Louis Reynaud, ainsi que la petite cinquantaine de stagiaires qui se sont relayés au chevet du glacier déclinant, et en particulier Florence Guirado.

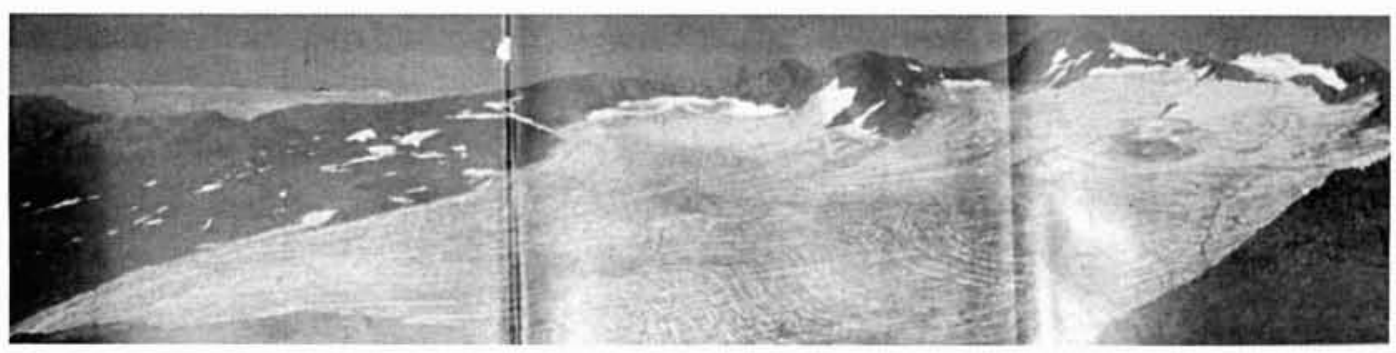

Le glacier de Sarennes en 1906. 


\section{LISTE DES TRAVAUX UNIVERSITAIRES RÉALISÉS SUR SARENNES}

Nous ne mentionnerons pas la traditionnelle communication présentée chaque année aux Journées de Glaciologie de la SHF à Grenoble, donnant le détail des mesures réalisées sur le glacier.

1975 COMPARAT Jacques. Observations glaciologiques sur le Glacier de Sarennes - saison 1974/1975. Publication à la Société Hydrotechnique de France -réunion des 17 et 18 mars 1976. Stage de 3ème année d'école d'Ingénieur INPG

1980 NEYRET Véronique et VALLA François. Variation saisonnière des densités sur le Glacier de Sarennes - Publication des mesures par station et par relevé 1961 - 1980. Stage (IUT de statistiques, Université Joseph Fourier Grenoble 1) ayant fait l'objet d'une communication pour le Société Hydrotechnique de France, section Glaciologie, 1980.

1984 BIENVENU Thierry. Le modèle linéaire appliqué au bilan de masse du glacier de Sarennes. Mémoire de DEA de Mécanique des Milieux Géophysiques et Environnement, Université Joseph Fourier - Grenoble I, Juin 1984

1987 GOUDAL Régis. Deux mois de mesures au Glacier de Sarennes. Compte rendu d'un stage effectué dans le cadre d'une initiative individuelle, septembre 87

1989 RUBY Stéphane. Evolution du Glacier de Sarennes sur la période 1975 - 1989. Mémoire de Maîtrise des Sciences et Techniques " Sciences de la Terre et de la Vie appliquées aux milieux de Montagne ". C.I.S.M., 73370 Le Bourget du Lac, Novembre 1989

1991 PICH François. Glacier de Sarennes, bilan de masse et approche hydrologique. Rapport de stage de la Maitrise des Sciences et Techniques " Sciences de la Terre et de la Vie appliquées aux milieux de Montagne ". C.I.S.M., 73370 Le Bourget du Lac, Septembre 1991

1992 PICH François. Etude sismique du Glacier de Sarennes. Rapport de stage de la Maîtrise des Sciences et Techniques "Sciences de la Terre et de la Vie appliquées aux milieux de Montagne ". Université de Savoie - Institut Savoisien de la montagne. Janvier - Février 1992

1992 TAIRRAZ Vincent. Le Glacier de Sarennes en 1992, Bilan de Masse du 44 ème cycle et analyse hydrologique. Rapport de stage de la Mấtrise des Sciences et Techniques "Sciences de la Terre et de la Vie appliquées aux milieux de Montagne ". C.I.S.M., 73370 Le Bourget du Lac Novembre 1992

1993 GIRARD Guilhem. Etude photogrammétrique du Glacier de Sarennes entre 1975 et 1991. Rapport de stage de la Maîtrise des Sciences et Techniques "Sciences de la Terre et de la Vie appliquées aux milieux de Montagne ". C.I.S.M., 73370 Le Bourget du Lac, Janvier 1993

1994 ESCANDE Sébastien. Etude Hydrologique de la Sarennes, Traitement de données d'une station hydrométrique. Compte-rendu d'activité, Maîtrise des Sciences et Techniques "Sciences de la Terre et de la Vie appliquées aux milieux de Montagne ". C.I.S.M., 73370 Le Bourget du Lac, Janvier 1994

1994 PIEDALLU Christian. Glacier de Sarennes : Etude quantitative de 1975 à 1993 Mémoire de Maîtrise des Sciences et Techniques " Sciences de la Terre et de la Vie appliquées aux milieux de Montagne ". C.I.S.M., 73370 Le Bourget du Lac Janvier 1994

1994 GAY Michel. Mesures Hydrométriques du torrent de Sarennes. Etude réalisée à la demande de la S.A.T.A. (Société d'Aménagement Touristique de l'Alpe d'Huez) et effectuée en collaboration avec la division Hydrologie - Hydraulique du CEMAGREF de Lyon. Juin 1994

1994 PIEDALLU Christian. Les variations du glacier de Sarennes de 1906 à 1993. Stage post-maitrise, septembre 1994

1994 BARBET Denis. Premiers Résultats Hydrologiques sur le Glacier de Sarennes. Mémoire de DEA Mécanique des Milieux Géophysiques et Environnement. Université Joseph Fourier - Grenoble I, Septembre 1994

1995 ROCHARD Valérie, Le Glacier de Sarennes : Un siècle et demi d'histoire et de documents. Mémoire de Maîtrise des Sciences et Techniques " Sciences de la Terre et de la Vie appliquées aux milieux de Montagne ". C.I.S.M., 73370 Le Bourget du Lac, Juillet - Août 1995

PIEDALlU Christian. Calcul des bilans annuels du Glacier de Sarennes. Validité de la localisation des stations de mesures. Rapport d'activité Octobre 1995
1996 COLLOMBET Marielle. Histoires de Perches. Rapport de stage de Maîtrise de Physique et Applications de I'Université Joseph Fourier Grenoble I, Janvier - Mars 1996

1996 PETEUIL Christophe. Premiers essais de comparaison des bilans hydrologique et glaciologique du bassin versant glaciaire de Sarennes. Rapport de stage. 2ème année d'école d'ingénieur ENSHMG Juin Septembre 1996

1997 GROJEANNE Marie. Etude de la répartition de la neige sur le bassin versant glaciaire de Sarennes. Rapport de stage de Maîtrise de Sciences Physiques mention "Physique et Applications " Université Joseph Fourier - Grenoble I Janvier - Mars 1997

1997 BIRONNEAU Didier. Reconstitution des variations de la surface d'un glacier à l'aide des bilans de masse. Mémoire de D.E.A. Structures et Dynamiques Spatiales, Institut de Géographie Alpine, Université Joseph Fourier - Grenoble I, Juin 1997

1997 PEILLEX Stéphanie. Etude Hydrologique de la Sarennes : Analyse des écoulements entre deux points de mesures. Rapport de stage de Maîtrise de Sciences Physiques, Université Joseph Fourier - Grenoble I, Avril - Juin 1997

1997 SIX Delphine. Analyse statistique de la série des bilans de masse glaciaires relevés sur différents glaciers voisins des Alpes françaises Mémoire de DEA Physico-Chimie de l'Atmosphère, Université Joseph Fourier-Grenoble I, Juin 1997

1997 PRADEAU Sophie. Mesures glaciologiques sur le Glacier de Sarennes et étude hydrologique (calcul du temps de transfert entre 3 stations de mesure). Rapport de stage de Licence de Mécanique, UFR de Mécanique - Université Joseph Fourier Grenoble I, Juin - Juillet 1997

1997 FAVIER Vincent. Résultats hydrologiques du bassin de Sarennes après 4 ans de mesures à l'exutoire. Rapport de stage. 2ème année école d'ingénieur ENSHMG (division hydrologie - hydraulique du Cemagref de Lyon et division nivologie du Cemagref de Grenoble) Juillet - Août 1997

1997 JOMELLI Vincent. Bilan Hydrologique du Glacier de Sarennes (1996 - 1997), Rapport de stage réalisé dans le cadre d'une étude post-doctorale. Novembre 1997

1998 POUGET Thibaut. Bilans et Mesures Météorologiques sur le Glacier de Sarennes. Rapport de stage de Maitrise de Sciences Physiques. Université Joseph Fourier - Grenoble I, Avril - Juin 1998

1998 JUVANON Philippe. Maintenance de l'Instrumentation sur le Bassin Versant de Recherche et d'Expérimentation de Sarennes. Rapport de stage de Licence de Sciences Physiques mention " Physique et Applications ", Université Joseph Fourier - Grenoble I, Mai - Juillet 1998

1998 GUIRADO Florence. Le Bassin Versant Glaciaire de Sarennes : Comparaison des Bilans Glaciologiques et Hydrologiques. Rapport de stage de DESS " Aménagement des Pays de Montagne " C.I.S.M. 73370 Le Bourget du Lac, Mai 1998

1998 FAVIER Vincent. Résultats des mesures sur le Glacier de Sarennes en vue de l'affinage des calculs hydrologiques et glaciologiques des bilans. Rapport de stage. Poursuite du stage de 2ème année école d'ingénieur ENSHMG, Juillet - Septembre 1998

1998 VERGNOLLE Nicolas. Superposition de photographies obliques sur un MNT pour l'identification de zones enneigées. Etude de faisabilité. Mémoire de DEA de Mécanique des Milieux Géophysiques et Environnement. Université Joseph Fourier - Grenoble I, Juin 1998

1999 JOURNET Nicolas. Bilans et recherche de gradients sur le Glacier de Sarennes. Rapport de stage de Maîtrise de Physique et Applications. Université Joseph Fourier - Grenoble 1. Avril - Juin 1999

1999 BOUTILLIER Benoît. Etude Préliminaire de la répartition spatiale des précipitations liquides sur le bassin versant glaciaire de Sarennes. Rapport de stage, 2ème année d'Ecole d'ingénieur, ENSHMG, Juillet Août 1999

1999 TORINESI Olivier. Corrélation des bilans de masse des glacier de Sarennes et Saint Sorlin avec les facteurs météorologiques et reconstitution de ces bilans à partir de 1923. Mémoire de DEA de Mécanique des Milieux Géophysiques et Environnement Université Joseph Fourier - Grenoble 1, Juin 1999

1999 DUROT Katia. Modélisation hydrologique distribuée du bassin versant nivo-pluvial de Sarennes. Validation des données d'entrée et développement d'un module de fonte nivale sous forêt. Thèse de doctorat au LTHE, Université Joseph Fourier - Grenoble I, Octobre 1999

2000 BONTRON Guillaume.Modélisation des débits à l'exutoire du Glacier de Sarennes et reconstitutions des années 1950 - 1990. Mémoire de DEA de Mécanique des Milieux Géophysiques et Environnement Université Joseph Fourier - Grenoble I. 


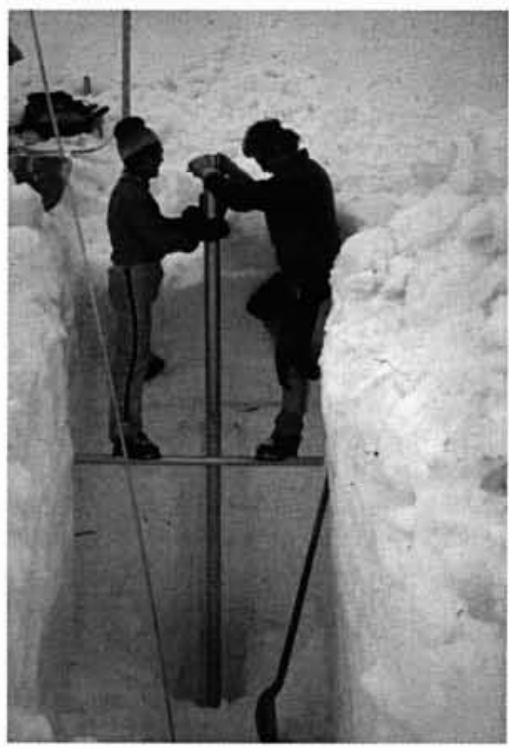

Carottage à l'ancienne, en 1975.

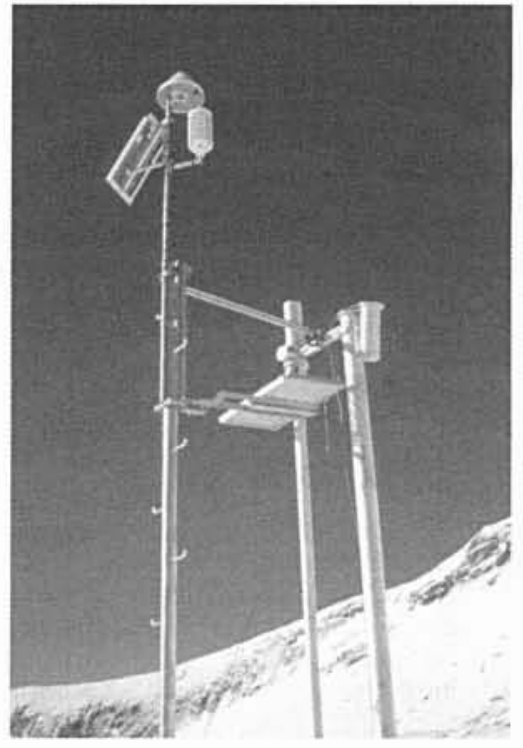

Télénivomètre EDF.

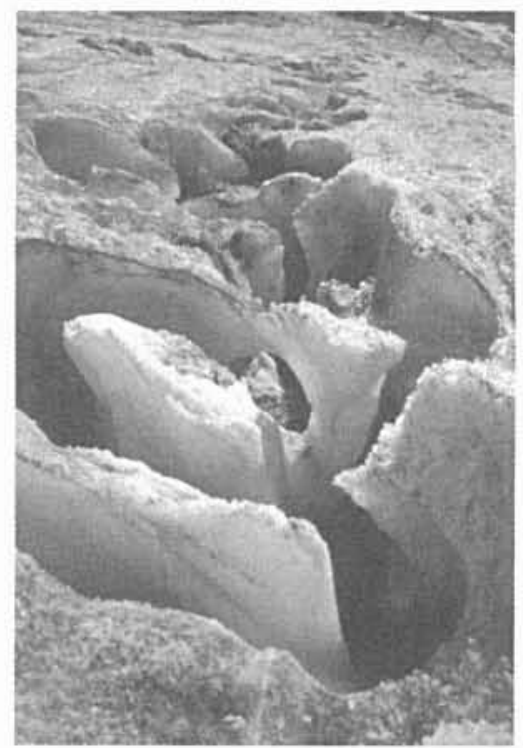

Bédière sur le glacier.

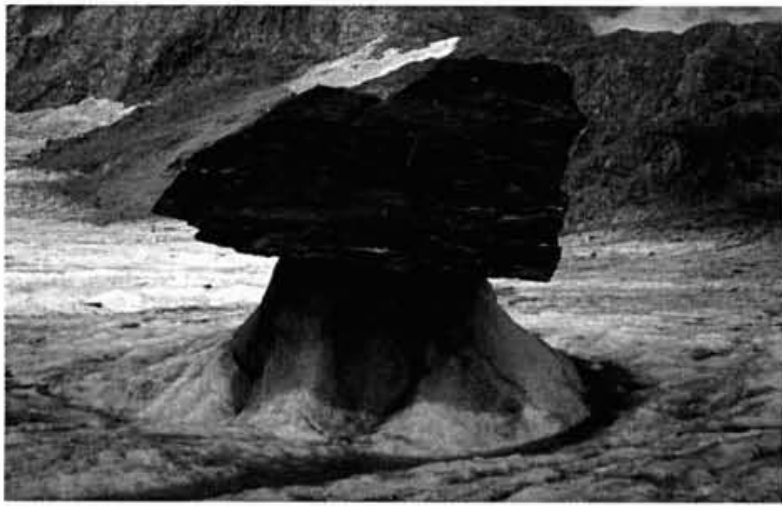

Table glaciaire.

\section{BIBLIOGRAPHIE}

[1] Flusin G., JACOB C. and OffNer J. - 1909 - " Etudes glaciaires, géographiques et botaniques dans le massif des Grandes Rousses. Rapport des campagnes de 1905 et 1906 " in Etudes Glaciologiques, vol I, p. 33-112 Ministère de I'Agriculture, Paris.

[2] Cherrey M. - 1951 - " Observations d'octobre 1949 à octobre $1950 \mathrm{du}$ glacier de Sarennes ". Mémoires et Travaux de la Section de Glaciologie de la SHF, La Houille Blanche, numéro spécial A. p. 3 à 10.

[3] VALLA F. - 1995 - " The mass balance of Glacier de Sarennes "Zeitschrift für Gletsherkunde und Glazialgeologie, 31, p. 189-197.

[4] Peteull. C.- 1996 - "Premiers essais de comparaison des bilans hydrologique et glaciologique du bassin versant glaciaire de Sarennes. Rapport de stage, Ecole Nationale Supérieure d'Hydraulique et de Mécanique de Grenoble.

[5] Francou B, Berton P, Wagnon P, Pouyaud B et Valdiviesco H 1997 - " Mesures météorologiques, hydrologiques et glaciologiques sur le glacier de Zongo, année hydrologique 1995-96 " Informe 55, OrstomBolovie.

[6] Bironneau D., - 1997 - " Reconstitution des variations de la surface d'un glacier à l'aide des bilans de masse ". Mémoire de DEA " Structures et Dynamiques ", IGA-Joseph Fourier, Grenoble.

[7] Valla F., Piedallu C., - 1997 - " Volumetric variations of Glacier de Sarennes, French Alps, during the two last centuries ". Annals of Glaciology, volume 24, p. $361-366$.

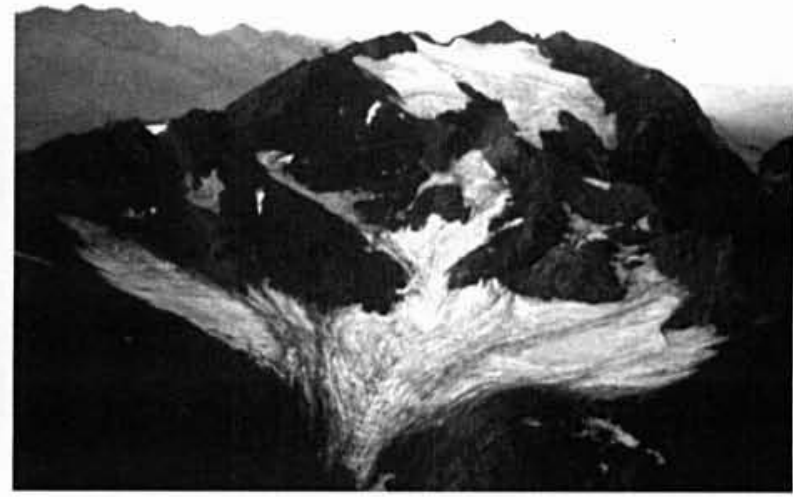

Vue aérienne du glacier en 1998.

[8] BARBet D- 1994 - " Premiers résultats hydrologiques sur le glacier de Sarennes " Mémoire de DEA " Mécanique des Milieux Géophysiques et Environnement " Cemagref-Lyon et Grenoble.

[9] Martin E., Lejeune Y., Leblois E., Valla F., Bironneau D., - 1997 "Modélisation hydrométéorologique de l'aléa de crue nivo-pluviale ". rapport final Contrat de Plan entre l'Etat et la Région Rhône-Alpes 1994-1998.

[10] Braun L., Reynaud L., Valla F. - 1993 - " Changes in snow and ice storage : measurement and simulation "Zürcher Geographische Schriften, Heft 53 seite $131-142$

[11] VinCENT C., VAllon M. - 1997 - " Meteorological controls on mas balance : empirical relations suggested by measurements on glacier de Sarennes, France ". Journal of Glaciology, Vol 43 Ne 143, p. 131-137.

[12] EsCher-Vetter H., Reinwarth O. - 1994 - " Zwanzig Jahre Abflussmessung an der Pegelstation Vernagtvbach (Otztaler Alpen) 1974-1993 " Rapport Kommission für Glaziologie der Bayerischen Akademie der Wissenschaften , München, 48 pages.

[13] Funk M.. Bósch H, VAlLa F. - 1993 - " Mesure des épaisseurs de glace par méthode radar au glacier de Sarennes " 45 e journées de Glaciologie de la SHF, février 1993, Grenoble 13 pages.

[14] GAY M.- 1994 - "Mesures Hydrométriques du Torrent de Sarennes, Estimation des débits d'eau " Etude Cemagref.

[15] Pradeau S.- 1997 - " Calcul du temps de transfert entre trois stations de mesures "Rapport de Stage, Licence de Mécanique, Université Joseph Fourier, Grenoble.

[16] VALLA F, - 1989 - " Forty years of mass balance, glacier de Sarennes " Congrès de Lom, Norvège, Annals of Glaciology vol. 13, p. 269-272. 